\title{
A CRITERION ON A SUBDOMAIN OF THE DISC FOR ITS HARMONIC MEASURE TO BE COMPARABLE WITH LEBESGUE MEASURE
}

\author{
A. L. VOLBERG
}

(Communicated by Palle E. T. Jorgensen)

\begin{abstract}
A subdomain $\mathscr{\theta}$ of the disc $\mathbb{D}$ is called a boundary layer if $\omega(\mathscr{O}, \cdot) \geq \alpha \cdot m$, where $\omega(\mathscr{O}, \cdot)$ is the harmonic measure of $\mathscr{O}$. The metric criterion in terms of $\partial \mathscr{O}$ is given for the case when $\alpha$ is near 1 .
\end{abstract}

\section{Statements}

Much interesting analysis has resulted from attempts to understand the structure of $P^{2}(\mu)$, the closure in $L^{2}(\mu)$ of the set $\mathscr{P}_{A}$ of all analytic polynomials, where $\mu$ is a positive finite Borel measure with compact support in the complex plane $\mathbb{C}$. The recent achievement in this field due to $J$. Thomson [4] asserts that $P^{2}(\mu) \neq L^{2}(\mu)$ if and only if there is a point $c$ such that

$$
|p(c)| \leq K\|p\|_{L^{2}(\mu)} \quad \forall p \in \mathscr{P}_{A} .
$$

(Such points are called points of bounded evaluation.) In other words Thomson has solved a problem of Mergelyan-Brennan. He even has achieved more: a description of $P^{2}(\mu)$ in terms of points of bounded evaluations.

But unfortunately, this does not help much when one is interested in a description of $P^{2}(\mu)$ in terms of $\mu$ itself.

Perhaps the best example is a so-called splitting problem. In this problem,

$$
d \mu=w(1-r) r d r d \theta+h(\theta) d m(\theta)=\mu_{\mathrm{D}}+\mu_{\mathrm{T}}
$$

is a sum of a radially symmetric measure $\mu_{\mathbb{D}}$ in the unit disc $\mathbb{D}$ and a measure $\mu_{\mathrm{T}}$ on $\mathbb{T}=\partial \mathbb{D}$. What are the conditions on the pair $(w, h)$ necessary and sufficient for the splitting:

$$
P^{2}(\mu)=P^{2}\left(\mu_{\mathbb{D}}\right) \oplus L^{2}\left(\mu_{\mathrm{T}}\right) ?
$$

This problem was solved in [5] and [6]. And, loosely speaking, the proof proceeds as follows. Assertion (**) can be reduced to a uniqueness theorem for

Received by the editors November 30, 1989.

1980 Mathematics Subject Classification (1985 Revision). Primary 31A10, 31B35, 31C15; Secondary $47 \mathrm{~B} 38,28 \mathrm{~A} 12$. 
functions $f \in C^{1}(\mathbb{D})$ such that $\left(\bar{\partial} \stackrel{\text { def }}{=} \frac{1}{2}\left(\frac{\partial}{\partial x}+i \frac{\partial}{\partial y}\right)\right)$

$$
|\bar{\partial} f(z)| \leq w(1-|z|)
$$

( $w$ is from $(*)$ ). Introducing the set $E_{w}$ of "singular values" of $f ; E_{w} \stackrel{\text { def }}{=}\{z \in$ $\mathbb{D}:|f(z)| \leq w(1-|z|)\}$, one can see that for suitable bounded $g$ the function $F \stackrel{\text { def }}{=} f e^{g}$ is holomorphic in $\Omega=\mathbb{D} \backslash E_{w}$.

Now the following problem arises naturally in the proof of the main result of [5] and [6]: How to characterize closed subsets $E$ of the unit disc $\mathbb{D}$ for which the domain $\mathbb{D} \backslash E$ (we suppose that $E$ does not split $\mathbb{D}$ ) is a boundary layer; i.e.,

$$
\omega(\mathbb{D} \backslash E, I, \zeta) \geq c|I|, \quad c>0,
$$

for all arcs $I, I \subset \mathbb{T}$ ? Here $|I|=m I$ is a normed Lebesgue measure of $I$.

Strictly speaking the $E$ in [5] and [6] are of a special kind. So one can succeed there without answering this general question. But it is natural to try to answer this question. And this is done to some extent in the present work.

Let $\mathscr{O}=\mathbb{D} \backslash E$. We suppose that $0 \in \mathscr{O}$ and denote $\omega(\mathscr{O}, \cdot, 0)$ by $\omega(\cdot)$. In what follows, $\mathscr{O}$ is a regular with respect to the Dirichlet problem; $P(a, \zeta)=$ $\left(1-|a|^{2}\right) /|1-\bar{a} \zeta|^{2}$ is the Poisson kernel, $a \in \mathbb{D}, \zeta \in \mathbb{T}, P^{\mu}(\zeta) \stackrel{\text { def }}{=} \int_{\mathbb{D}} P(a, \zeta) d \mu(a)$ for a measure $\mu$ in $\mathbb{D}$.

It is clear that $\mathscr{O}$ is a boundary layer if and only if

$$
P^{\omega}(\xi) \leq q<1, \quad \xi \in \mathbb{T} .
$$

When this case occurs we will say that $\mathscr{O}$ is a $(1-q)$-boundary layer. In fact, (1.1) means that $\omega(I) \geq(1-q) \cdot|I|$ for all $I$.

Surely $P^{\omega} \leq 1$ for an arbitrary $\mathscr{O}$, and $P^{\omega}<1$ a.e. with respect to $m$ if and only if the measure $\omega \mid \mathbb{T}$ and $m$ are mutually absolutely continuous.

The metric criterion in terms of capacity for $E$ to satisfy (1.1) will be given here in the following two cases:

(1) if $q$ is less than a certain absolute constant;

(2) if $E$ lies in a Stoltz domain;

$$
\Gamma\left(e^{i \theta}\right) \stackrel{\text { def }}{=}\left\{a \in \mathbb{D}:\left|e^{i \theta}-a\right| \leq C(1-|a|)\right\}, \quad C<\infty .
$$

To state the results we need some notation. Let $\mathscr{F}=\{I\}$ be the collection of all the dyadic arcs of $T, Q_{I}$ be a square in $\mathbb{D}$ based on $I, T Q_{I}$ be its top half, and $C_{I}$ be the center of $T Q_{I}$. For a subset $e$ of $Q_{I}$ we denote by $\lambda e$ the image of $e$ under the mapping $z \rightarrow \lambda\left(z-C_{I}\right)+C_{I}$.

1.1. Proposition. Let $1 / 2 \mathbb{D} \subset \mathcal{O}$. Then for an absolute $A_{1}$

$$
P^{\omega}(\xi) \leq A_{1} \sum_{I \in \mathscr{F}}\left|\frac{1-\left|C_{I}\right|}{1-\bar{C}_{I} \xi}\right|^{2} \operatorname{cap}\left(\frac{E \cap T Q_{I}}{2|I|}\right), \quad \forall \xi \in T .
$$

In particular,

$$
\sup _{\xi \in T} \sum_{I \in \mathscr{F}}\left|\frac{1-\left|C_{I}\right|}{1-\bar{C}_{I} \xi}\right|^{2} \operatorname{cap}\left(\frac{E \cap T Q_{I}}{2|I|}\right) \leq \frac{q}{A_{1}}
$$

implies (1.1). 
An estimate from below for $P^{\omega}(\xi)$ is replaced by the following proposition.

1.2. Proposition. There exist absolute constants $A_{2}$ and $q_{0}>0$ such that if (1.1) holds with $q \leq q_{0}$, then

$$
\sup _{\xi \in T} \sum_{I \in \mathscr{F}}\left|\frac{1-\left|C_{I}\right|}{1-\bar{C}_{1} \xi}\right|^{2} \operatorname{cap}\left(\frac{E \cap T Q_{1}}{2|I|}\right) \leq A_{2} q .
$$

For $E$ in a Stoltz domain

$$
\sum_{I \in \mathscr{F}} \cdots \leq A_{2} P^{\omega}(\xi), \quad \xi \in T .
$$

The following result follows immediately.

1.3. Theorem. If $E$ is in a Stoltz domain, then the following assertions are equivalent:

(1) $\mathscr{O}$ is a boundary layer;

(2) $\sup _{\xi \in T} \sum_{I \in \mathscr{F}}\left|\frac{1-\left|C_{I}\right|}{1-\bar{C}_{1} \xi}\right|^{2} \operatorname{cap}\left(\frac{E \cap T Q_{I}}{2|I|}\right)<\infty$; and

(3) $\sum_{I \in \mathscr{F}} \operatorname{cap}\left(\frac{E \cap T Q_{I}}{2|I|}\right)<\infty$.

For a domain $\mathscr{O}=\mathbb{D} \backslash E$, let us consider domains $\mathscr{O}_{n}=\mathscr{O} \cup(1-1 / n) \mathbb{D}$, $\omega_{n}(\cdot)=\omega\left(\mathscr{O}_{n}, \cdot, 0\right)$. It is worthwhile to note that there is a criterion which guarantees that $\mathscr{O}_{n}$ is a $\left(1-\varepsilon_{n}\right)$-boundary layer with $\lim \varepsilon_{n}=0$. In this case, we call $\mathscr{O}$ a good boundary layer.

1.4. Theorem. $\mathscr{O}$ is a good boundary layer if and only if the series

$$
\sum_{I \in \mathscr{F}}\left|\frac{1-\left|C_{I}\right|}{1-\bar{C}_{I} \xi}\right|^{2} \operatorname{cap}\left(\frac{E \cap T Q_{I}}{2|I|}\right)
$$

converges uniformly on $T$.

We conclude this section with one more notation:

$$
\nu_{E} \stackrel{\text { def }}{=} \sum_{I \in \mathscr{F}}|I| \operatorname{cap}\left(\frac{E \cap T Q_{I}}{2|I|}\right) \delta_{C_{I}},
$$

where $\delta_{C_{I}}$ is the point measure. The proof of Proposition 1.2 is rather amusing but we begin with the following instead.

\section{The PROOF OF Proposition 1.1}

It is sufficient to verify that

$$
\omega\left(T Q_{I}\right) \leq A|I| \operatorname{cap}\left(\frac{E \cap T Q_{I}}{2|I|}\right) .
$$

We show that for large absolute constant $B$ there is a rectangle $R$ with base containing $I$, contained in $2 I$, such that $\mathbb{D} \cap \partial R \subset Q_{2 I} \backslash Q_{I}$, and

$$
\omega\left(\mathscr{O}, E \cap T Q_{I}, z\right) \leq B \operatorname{cap}\left(\frac{E \cap T Q_{I}}{2|I|}\right), \quad \forall z \in \mathbb{D} \cap \partial R .
$$


It follows from the maximum principle that it is sufficient to find a closed contour $R_{1}$ surrounding $E_{I} \stackrel{\text { def }}{=} E \cap T Q_{I}$ with property (2.2) and such that $R_{1} \subset$ $\frac{3}{2} T Q_{I}$. Let $E_{I}^{*}$ be the image of $E_{I}$ under the conformal mapping $\varphi: \mathbb{D} \rightarrow \frac{1}{4} \mathbb{D}$ such that $\varphi\left(C_{I}\right)=0$. It is clear that

$$
a_{1} \operatorname{cap} E_{I}^{*} \leq \operatorname{cap}\left(E_{I} / 2|I|\right) \leq a_{2} \operatorname{cap} E_{I}^{*},
$$

where $0<a_{1}, a_{2}<\infty$ do not depend on $I$. Now we apply Lemma 6.1 (see §) to $E_{I}^{*}, \frac{1}{4} \mathbb{D}$. Note that $E_{I}^{*} \subset \mathbb{D} / 4 \lambda, \varphi\left(\frac{3}{2} T Q_{I}\right) \subset \mathbb{D} / 4 \lambda^{\prime}$ where $\lambda>\lambda^{\prime}>1$ and $\lambda, \lambda^{\prime}$ do not depend on $I$. Assertion (6.3) shows that $R_{I}=\varphi^{-1}\left(T / 4 \lambda^{\prime \prime}\right)$ suits us for some $\lambda^{\prime \prime} \in\left(\lambda^{\prime}, \lambda\right)$.

An application of the Poisson formula in $\mathscr{O} \backslash$ clos $R$ to the function $\omega\left(\mathscr{O}, E_{I}, z\right)$, harmonic in this domain, gives us $(0 \in \mathscr{O} \backslash \operatorname{clos} R$ as $1 / 2 \mathbb{D} \subset \mathscr{O})$ :

$$
\omega\left(\mathscr{O}, E_{I}, 0\right) \leq 4 B|I| \operatorname{cap}\left(E_{I} / 2|I|\right) \text {. }
$$

\section{The PROOF OF Proposition 1.2}

Let $\widetilde{T Q}_{I}$ denote the union of $T Q_{I}$ and all $T Q_{J}$ neighboring $T Q_{I}$.

We suppose that $(1.1)$ is valid with $q$ sufficiently small. We will show that under this assumption the inequality

$$
\omega\left(\widetilde{T Q_{I}}\right) \geq a|I| \operatorname{cap}\left(\frac{E \cap T Q_{I}}{2|I|}\right)
$$

holds. Inequality (3.1) is the reverse of (2.1). It is also clear that it may hold only for sufficiently small $q$.

It follows from (3.1) and Harnack's inequality that

$$
P^{\nu_{E}}(\xi) \leq A a^{-1} P^{\omega}(\xi), \quad \xi \in T,
$$

which proves Proposition 1.2.

Inequality (3.1) is a stronger version of Lemma 6.2. To prove (3.1), we put $u_{1}(z)=\omega\left(\mathscr{O}, E \cap \widetilde{T Q_{I}}, z\right), u_{2}(z)=\operatorname{cap}\left(\frac{E \cap T Q_{I}}{2|I|}\right) \cdot \omega\left(\mathscr{O}, \frac{1}{4} \Gamma, z\right)$ and compare these functions on a specially built contour $\gamma, \gamma \subset Q_{I} \backslash Q_{1 / 2 I}$, surrounding $E_{I}=E \cap T Q_{I}$. From the left part of (6.2), it follows that

$$
\begin{aligned}
& u_{1}(z) \geq \omega\left(\widetilde{T Q}_{I} \backslash E, E, z\right) \\
& \geq \omega\left(\widetilde{T Q_{I}} \backslash E_{I}, E_{I}, z\right) \geq a u_{2}(z), \quad z \in \partial T Q_{I} .
\end{aligned}
$$

We find two curves $\gamma_{1}, \gamma_{2}$ connecting $\partial T Q_{I}$ with $\mathbb{T}$ and such that $\gamma_{1}, \gamma_{2}$ and a part of $\partial T Q_{I}$ from a contour $\gamma$ separating $1 / 2 I$ from 0 . We have to find $\gamma_{1}, \gamma_{2}$ in such a way that the left part of (3.3) is not less than the right part of (3.3) on $\gamma$. If it is the case, (3.1) will follow, as then

$$
u_{1}(0) \geq a u_{2}(0) \geq a \cdot \operatorname{cap}\left(\frac{E_{I}}{2|I|}\right)|I|
$$

by virtue of the condition of our proposition. 
Now let $R_{1}, R_{2}$ be rectangles which form $Q_{I} \backslash\left(T Q_{I} \cup Q_{1 / 2 I}\right)$. We will find $\gamma_{i} \subset R_{i}, i=1,2$.

It is obvious that

$$
u_{2}(z) \leq A \operatorname{cap}\left(\frac{E_{I}}{2|I|}\right) \cdot \frac{1-|z|}{|I|}, \quad z \in R_{1} \cup R_{2} .
$$

The Green function of $\mathscr{O}=\mathbb{D} \backslash E$ will be denoted by $G_{\mathscr{O}}(a, b)$. Let $G(a, b)=\log \left|\frac{1-\bar{a} b}{a-b}\right|$. We will use the notation:

$$
G_{\mathscr{O}}(\zeta) \stackrel{\text { def }}{=} G_{\mathscr{O}}(\zeta, 0), \quad G(\zeta) \stackrel{\text { def }}{=} \log 1 /|\zeta|=G(0, \zeta)=G(\zeta, 0) .
$$

Our first step will result in proving the implication

$$
G_{\mathscr{Q}}(z) \geq\left(1-\varepsilon_{0}\right) G(z) \Rightarrow u_{1}(z) \geq a \cdot \operatorname{cap}\left(\frac{E_{I}}{2|I|}\right) \cdot \frac{1-|z|}{|I|},
$$

where $\varepsilon_{0}$ is a certain positive absolute constant.

Combining (3.4) and (3.5) we see that it remains to be proven that for $i=$ 1,2 there is a curve $\gamma_{i}$ in $R_{I}$ connecting the top side of $R_{i}$ with its bottom side such that

$$
G_{\mathscr{Q}}(z) \geq\left(1-\varepsilon_{0}\right) G(z)
$$

on $\gamma_{i}$.

Step 1 . Let $R$ denote a rectangle in $\mathbb{D}$ based on $2 I$ and $1 / 2|I|$ in height and let $u(z) \stackrel{\text { def }}{=} \omega\left(R \backslash E, l_{I}, z\right)$ where $l_{I}$ is the middle half of the top side of $R$.

It follows from (3.3) that

$$
u_{1}(z) \geq a \cdot u(z) \operatorname{cap}\left(\frac{E_{I}}{2|I|}\right), \quad z \in R .
$$

Let $u_{0}(z) \stackrel{\text { def }}{=} \omega\left(R, l_{I}, z\right)$. It is clear that

$$
z \in R_{1} \cup R_{2} \Rightarrow b \frac{1-|z|}{|I|} \leq u_{0}(z) \leq B \frac{1-|z|}{|I|},
$$

with absolute $b, B, 0<b<B<\infty$. It follows from (3.7) and (3.8) and (3.5) is implied by the assertion

$$
z \in R_{1} \cup R_{2}, \quad G_{\mathscr{O}}(z) \geq\left(1-\varepsilon_{0}\right) G(z) \Rightarrow u(z) \geq a u_{0}(z) .
$$

The Poisson formula in $R \backslash E$ gives:

$$
\begin{aligned}
u(z) & =u_{0}(z)-\int_{E \cap R} u_{0}(\zeta) d \omega(R \backslash E, \zeta, z) \\
& \geq b \frac{1-|z|}{|I|}-B \int_{E \cap R} \frac{1-|\zeta|}{|I|} d \omega(R \backslash E, \zeta, z) \\
& \geq b \frac{1-|z|}{|I|}-\frac{B}{|I|} \int_{E \cap R} \log \frac{1}{|\zeta|} d \omega(\mathscr{O}, \zeta, z) \\
& =b \frac{1-|z|}{|I|}-\frac{B}{|I|} \int_{E \cap R} G(\zeta) d \omega(\mathscr{O}, \zeta, z) .
\end{aligned}
$$


Applying the Poisson formula in $\mathscr{O}$ to the positive harmonic function $G(\zeta)-$ $G_{\mathcal{O}}(\zeta)$, we get

$$
\int_{E} G(\zeta) d \omega(\mathscr{O}, \zeta, z)=G(z)-G_{\mathscr{O}}(z) \leq \varepsilon_{0} G(z) \leq A \varepsilon_{0}(1-|z|) .
$$

Combining this with (3.10), we get

$$
u(z) \geq \frac{1-|z|}{|I|}\left(b-\varepsilon_{0} B A\right) \geq \frac{b}{2} B^{-1} \cdot u_{0}(z)
$$

if $\varepsilon_{0} \leq \frac{1}{2} b / B A$. So (3.9) and at the same time (3.5) are proved. Note that $\varepsilon_{0}=1 / 50$ suits us well.

Step 2. The assertion that $P^{\omega}(\xi) \leq q_{0}$ with small $q_{0}$ has not been used yet (we have used only that $q_{0}<1$ ). Here the smallness of $q_{0}$ will play its part. We will prove the following implication:

$$
\begin{aligned}
& P_{\omega}(\xi) \leq q_{0}, \\
& \xi \in T \Rightarrow \forall I \in \mathscr{F} \operatorname{cap}\left(\frac{\left\{z \in T Q_{I}: G_{\mathscr{O}}(z) \leq\left(1-q_{0}^{1 / 4}\right) G(z)\right\}}{2|I|}\right) \leq A q_{0}^{1 / 4} .
\end{aligned}
$$

We see that (3.6) follows immediately from (3.12) and (6.1).

To prove (3.12), let us fix a point $c$ on $T, \Gamma_{c, \delta} \stackrel{\text { def }}{=}\left\{z: z=c+\delta e^{i \theta}, 0 \leq\right.$ $\theta<2 \pi\}$. The function $G_{\mathscr{O}}(z) \stackrel{\text { def }}{=} G_{\mathscr{O}}(z)(z \in \mathscr{O}) \stackrel{\text { def }}{=} 0(z \notin \mathscr{O})$ is subharmonic in int $\Gamma_{c, \delta}$ for $\delta \leq 1 / 2$ and $\Delta G_{\mathscr{Q}}=\omega$ in the distribution theory sense. Let $\mu_{\mathscr{O}}(t)=\omega(\{z:|z-c|<t\})$. The application of the Jensen formula to $G_{\mathscr{O}}$ in int $\Gamma_{c, \delta}$ gives

$$
\int_{0}^{\delta} \frac{\mu_{0}(t)}{t} d t=\frac{1}{2 \pi} \int_{0}^{2 \pi} G_{\mathscr{O}}\left(c+\delta e^{i \theta}\right) d \theta=\frac{1}{2 \pi} \int_{\Gamma_{i, \delta}} G_{\mathcal{O}}(z) \frac{|d z|}{\delta} .
$$

By the condition of Proposition 1.2 for all $c, \delta, t<\delta$ we have

$$
\mu_{\mathscr{O}}(t) \geq\left(1-q_{0}\right) \mu(t) \text {, }
$$

where $\mu(t) \stackrel{\text { def }}{=} m(\{z:|z-c|<t\})$. The analogue of (3.13) with the subscript $\mathscr{O}$ skipped takes place. This and (3.13) show that

$$
\frac{1-q_{0}}{2 \pi} \int_{\Gamma_{c, \delta}} G(z) \frac{|d z|}{\delta} \leq \frac{1}{2 \pi} \int_{\Gamma_{c, \delta}} G_{\mathscr{O}}(z) \frac{|d z|}{\delta},
$$

i.e.,

$$
\int_{\Gamma_{c, \delta}}\left(G(z)-G_{\mathscr{O}}(z)\right) \frac{|d z|}{2 \pi \delta} \leq q_{0} \int_{\Gamma_{c, \delta}} G(z) \frac{|d z|}{2 \pi \delta} .
$$

Taking into account that $G(z) \leq A \delta$ on $\Gamma_{c, \delta}, G\left(c+\delta e^{i \theta}\right) \geq \delta / 2$ for $\theta \epsilon$ $(\pi / 3,2 \pi / 3)$, we get from $(3.15)$

$$
m\left\{\theta \in\left(\frac{\pi}{3}, \frac{2 \pi}{3}\right): 1-\frac{G_{\mathscr{O}}\left(c+\delta e^{i \theta}\right)}{G\left(c+\delta e^{i \theta}\right)} \geq q_{0}^{1 / 2}\right\} \leq A q_{0}^{1 / 2} .
$$


Set $F_{I} \stackrel{\text { def }}{=}\left\{z \in T Q_{I}: G_{\mathscr{O}}(z) \leq\left(1-q_{0}^{1 / 4}\right) G(z)\right\}$. This set is closed, as $G_{\mathscr{O}}$ is a continuous function. The function $G_{\mathscr{Q}}-G$ is subharmonic in $Q_{2 I} \backslash F_{I}$; $G_{\mathscr{O}}-G \leq 0$ on $\partial\left(Q_{2 I} \backslash F_{I}\right)$ and

$$
G_{\mathscr{O}}-G \leq-a \cdot q_{0}^{1 / 4}|I| \quad \text { on } F_{I} .
$$

Now we choose $c$ to be the center of $I, \delta=3 / 4|I|, z_{0}=c+\delta e^{i \theta_{0}}, \theta_{0} \in$ $(\pi / 3,2 \pi / 3)$. Then it follows from (3.17) that

$$
G_{\mathscr{O}}\left(z_{0}\right) \leq-a \cdot q_{0}^{1 / 4}|I| \omega\left(Q_{2 I} \backslash F_{I}, F_{I}, z_{0}\right) .
$$

At the same time point $z_{0}$ can be chosen in such a way (provided that $q_{0}$ is sufficiently small: see (3.16)) that

$$
G_{\mathscr{O}}\left(z_{0}\right)-G\left(z_{0}\right) \geq-q_{0}^{1 / 2} G\left(z_{0}\right) \geq-2 q_{0}^{1 / 2}|I| .
$$

It follows from (3.18) and (3.19) that

$$
\omega\left(Q_{2 I} \backslash F_{I}, F_{I}, z_{0}\right) \leq 2 a^{-1} \cdot q_{0}^{1 / 4},
$$

and (3.12) follows from this and the left part of (6.2).

\section{THE PROOF OF THEOREM 1.3}

Let $E$ lie in a Stoltz domain $\Gamma\left(\xi_{0}\right)=\left\{a \in \mathbb{D}:\left|\xi_{0}-a\right| \leq c_{\Gamma}(1-|a|)\right\}$. The function $P^{\nu_{E}}$ is lower-semicontinuous, and so

$$
\sup _{\mathbb{T}} P^{\nu_{E}}(\xi)=\operatorname{ess} \sup _{\mathbb{T}} P^{\nu_{E}}(\xi)=P^{\nu_{E}}\left(\xi_{0}\right)=\sum_{I \in \mathscr{F}} \operatorname{cap}\left(\frac{E_{I}}{2|I|}\right) .
$$

Therefore, (2) is equivalent to (3).

To prove $(1) \Rightarrow(3)$, let us denote by $I_{n}$ an arc of length $2^{-n}$ centered at $\xi_{0}, Q_{n}=Q_{I_{n}}$. Then $\sum_{n \geq 0} 2^{n} \omega\left(Q_{n+1} \backslash Q_{n}\right) \leq A\left(c_{\Gamma}\right) P^{\omega}\left(\xi_{0}\right)<\infty$. So the series $\sum_{n \geq 0} 2^{n} \omega\left(Q_{n}\right)$ converges. It remains to apply Lemma 6.2 (or the stronger assertion (3.1)) and take into account that $\omega\left(I_{n}\right) \geq c \cdot 2^{-n}$.

On the other hand, if (3) takes place and $E \subset \Gamma\left(\xi_{0}\right)$, then (1.5) is valid and $\mathscr{O}$ is a good boundary layer (see Theorem 1.4).

\section{THE PROOF OF THEOREM 1.4}

We put $E_{2^{m}}=\mathbb{D} \backslash \mathscr{O}_{2^{m}}, \quad \nu_{2^{m}}=\sum_{I \in \mathscr{F},|I| \leq 2^{-m}}|I| \operatorname{cap}\left(E_{I} / 2|I|\right) \delta_{c_{I}}$. Proposition 1.1 shows that

$$
P^{\omega_{2^{m}}}(\xi) \leq A_{1} \cdot P^{\nu_{2} m}(\xi), \quad \xi \in T,
$$

and so by virtue of (1.5), $\lim \left\|P^{\omega_{2^{m}}}\right\|_{\infty}=0$ which means that $\mathscr{O}$ is a good boundary layer.

Let $\mathscr{O}$ be a good boundary layer. Then $\left\|P^{\omega_{2^{m}}}\right\|_{\infty} \rightarrow 0$. Now Proposition 1.2 shows that $\left\|P^{\nu_{2^{m}}}\right\|_{\infty} \rightarrow 0$.

Surely a good boundary layer is a boundary layer. The converse is true sometimes (e.g., if $E=\mathbb{D} \backslash \mathscr{O}$ lies in a Stoltz domain) but is not true in general. 
V. I. Vasyunin called attention to the following:

5.1. Example. Let $D_{n}=D\left(r_{n} e^{i 2^{-n}}, \delta\left(1-\Gamma_{n}\right)\right)$, where $\delta$ is a fixed small constant, $E=\cup_{n \geq 0} D_{n}$. An inductive procedure permits us to choose $\left\{r_{n}\right\}$ converging to 1 so rapidly that $P^{\nu_{E}}(\xi) \leq q(\delta), \xi \in T$, and $\lim _{\delta \rightarrow 0} q(\delta)=0$. But (1.5) is obviously false.

5.2. Remark. Let $E \subset \Gamma\left(\xi_{0}\right)$. It is clear that the thinness of $E$ (in the sense of Wiener) guarantees that $\mathbb{D}=\mathscr{O} \backslash E$ is a boundary layer. In fact, the Wiener criterion of thinness is $\sum n \operatorname{cap}\left(E \cap A_{n}\left(\xi_{0}\right)\right)<\infty$ where $A_{n}=\left\{z: 2^{-n-1} \leq\right.$ $\left.\left|z-\xi_{0}\right| \leq 2^{-n}\right\}$. And (3) of Theorem 1.3 can be rewritten as $\sum \operatorname{cap}\left(\frac{E \cap A_{n}\left(\xi_{0}\right)}{2^{-n+1}}\right)<$ $\infty$. It remains to use the fact that $\operatorname{cap}(E / \varepsilon)=\operatorname{cap} E /(1+\log \varepsilon \cdot \operatorname{cap} E)$. In the simplest case $E=\bigcup D_{n}, D_{n}=D\left(r_{n}, \rho_{n}\right), \rho_{n}<2^{-n}, 1-r_{n} \sim 2^{-n}$, the domain $\mathcal{O}$ is a (good) boundary layer if and only if

$$
\sum_{n}\left(\log \frac{1-r_{n}}{\rho_{n}}\right)^{-1}<\infty
$$

\section{AUXILIARY ASSERTIONS}

For the sake of completeness, we state in this section some standard and well-known assertions that were used above.

Recall that the capacity of a compact $E$ is defined by the equality

$$
1 / \operatorname{cap} E=\lim _{z \rightarrow \infty}(g(z)-\log |z|),
$$

where $g$ is the Green function of $\mathbb{C} \backslash E$ with pole at $\infty$. We are always in a situation where $g$ is contained in a disc of radius $3 / 4$ in which case cap $E \subset$ $\left[0,(\log 4 / 3)^{-1}\right]$, and cap $E$ may also be described as

$\operatorname{cap} E=\sup \left\{\mu(E): \mu\right.$ is a positive measure supported on $E$ with $\left.U^{\mu} \leq 1\right\}$, where $U^{\mu}(z)=\int \log \frac{1}{|z-\zeta|} d \mu(\zeta)$ is the logarithmic potential.

We define the 1-dimensional Hausdorff content of a set by

$$
h_{1}(E)=\inf \left\{\sum r_{i}: E \subset \cup D\left(z_{i}, r_{i}\right), z_{i} \in \mathbb{C}\right\} \text {. }
$$

It is well known [2] that

$$
h_{1}(E) \leq A e^{-1 / \operatorname{cap} E}
$$

We have used the following

6.1 . Lemma. Let $Q$ be a square or a disc of diameter $d(Q)$ and $E \subset Q$ be a compact. If $\lambda>1$ then

$$
a_{\lambda} \operatorname{cap}\left(\frac{E}{2 d(Q)}\right) \leq \inf _{\zeta \in Q} \omega(\lambda Q \backslash E, E, \zeta) \leq a_{\lambda}^{-1} \operatorname{cap}\left(\frac{E}{2 d(Q)}\right) .
$$

Also if $\zeta \in \frac{1+\lambda}{2} Q$ with $\operatorname{dist}(\zeta, E) \geq b \cdot d(Q)$ then

$$
a_{\lambda, b} \operatorname{cap}\left(\frac{E}{2 d(Q)}\right) \leq \omega(\lambda Q \backslash E, E, \zeta) \leq a_{\lambda, b}^{-1} \operatorname{cap}\left(\frac{E}{2 d(Q)}\right) .
$$

The proof can be obtained by the comparison of the Green potential for $Q$ and the logarithmic potential of the equilibrium measure of $E$. 
6.2. Lemma. $\omega\left(\mathscr{O}, E \cap Q_{2 I}, 0\right) \geq a \cdot \operatorname{cap}\left(E_{I} / 2|I|\right)$.

Proof. Consider $u_{1}(z)=\omega\left(\mathscr{O}, E \cap Q_{2 I}, z\right), u_{2}(z)=\operatorname{cap}\left(\frac{E_{I}}{2|I|}\right) \cdot \omega(\mathscr{O}, 1 / 2 I, z)$. These functions are harmonic in $\mathscr{O}$. Then we compare $m$ on $\mathbb{D} \cap \partial Q_{I}$.

The left estimate in (6.2) implies that

$$
u_{1}(z) \geq \omega\left(Q_{2 I} \backslash E, E, z\right) \geq \omega\left(Q_{2 I} \backslash E_{I}, E_{I}, z\right) \geq a \cdot u_{2}(z)
$$

if $z \in \partial T Q_{I}$. If $z \in \partial Q_{I} \backslash \partial T Q_{I}$ then obviously

$$
u_{2}(z) \leq A \cdot \operatorname{cap}\left(\frac{E_{I}}{2|I|}\right) \cdot \frac{1-|z|}{|I|} .
$$

Our aim is to show that

$$
u_{1}(z) \geq a \cdot \operatorname{cap}\left(\frac{E_{I}}{2|I|}\right) \cdot \frac{1-|z|}{|I|}
$$

on $\partial Q_{I} \backslash\left(I \cup \partial T Q_{I}\right)$. Let $R$ be a rectangle in $\mathbb{D}$ based on $2 I$ and $\frac{1}{2}|I|$ in height. Let $u(z) \stackrel{\text { def }}{=} \omega(R, \partial R \cap \partial T Q I, z)$. Using the left part of (6.2) once again, we get for $z \in \partial R \cap \partial T Q_{I}$

$$
u_{1}(z) \geq \omega\left(Q_{2 I} \backslash E_{I}, e_{I}, z\right) \geq a \cdot \operatorname{cap}\left(E_{I} / 2|I|\right) u(z) .
$$

This inequality is valid in $R$, as $u_{1}$ is superharmonic in $R$ and positive. At the same time $u(z) \geq a \cdot \frac{1-|z|}{|I|}$ for $z \in R \cap \partial Q_{I}$. Thus $u_{1}(z) \geq a \cdot u_{2}(z)$ on $\mathbb{D} \cap \partial Q_{I}$. On $\partial \mathscr{O} \backslash Q_{I}, u_{2}=0, u_{1} \geq 0$. So $u_{1}(0) \geq a \cdot u_{2}(0)$.

It is interesting to compare Lemma 6.2 with (3.1). Assertion (3.1) is stronger, but it is valid if $\mathscr{O}$ is a $(1-q)$-boundary layer with a small $q$. As to the inequality in Lemma 6.2 , it is always true.

We conclude with two questions.

Question 1. Is any boundary layer the intersection of a finite collection of $(1-q)$ boundary layers with small $q$ ?

One can consider domains $\mathscr{O}$ of the sort $\mathscr{O}=\mathbb{R}_{+}^{n} \backslash E, E$ being a compact.

Question 2. What are the analogues of Propositions 1.1 and 1.2 for $n \geq 3$ ?

The diligent reader may suspect that the boundary layer problem has many points in common with problems concerning minimally thin and rarified sets. This is just the case. The article [3] treats these connections and contains references.

\section{REFERENCES}

1. A. A. Borichev and A. L. Volberg, Uniqueness theorems for almost analytic functions, Algebra and Analysis 1 (1989), 146-177. (Russian)

2. L. Carleson, Selected problems on exceptional sets, Van Nostrand, 1967.

3. M. Essèn, On minimal thinness, reduced functions and Green potentials, Upp. Univ. Dep. Math., no. 13, 1989.

4. J. E. Thomson, Approximation in the mean by polynomials, Ann. of Math. (2) (to appear). 
5. A. L. Volberg, The logarithm of an almost analytic function is summable, Soviet Math. Dokl. 26 (1982), 238-243.

6. A. L. Volberg and B. Joricke, Summability of the logarithm of an almost analytic function and a generalizations of the Levinson-Cartwright theorem, Mat. Sb. (N.S.) 130 (1986), 335348; English transl.: Math. USSR-Sb. 58 (1987), 337-349.

Leningrad Department, Steklov Mathematical Institute, USSR ACademy of Sciences, LENINGRAD, 191011

Current address: Department of Mathematics, University of Kentucky, Lexington, Kentucky 40506 